\title{
Myocardial fibrosis combined with NT-proBNP improves the accuracy of survival prediction in ADHF patients
}

\author{
Yiling Yao ${ }^{1}$, Li Feng ${ }^{1 *}$, Yanxiang Sun ${ }^{1}$, Shifei Wang ${ }^{2}$, Jie Sun ${ }^{1}$ and Bing $\mathrm{Hu}^{1}$
}

\begin{abstract}
Background: Soluble suppression of tumorigenesis-2 (sST2), Procollagen Type III N-Terminal Peptid (PIIINP) and $\mathrm{N}$-terminal pro-B-type natriuretic peptide (NT-proBNP) have been verified their role in predicting survival in acutely decompensated heart failure (ADHF). However, whether their combination could improve more specific and sensitive prognostic information than NT-proBNP alone remains unclear.

Methods: This was a prospective study, in which $217 \mathrm{ADHF}$ patients at admission were enrolled from November 2018 and August 2019 (mean age 66.18 years \pm 13.60,63.98\% male). The blood samples were collected to measure the concentrations of NT-proBNP, sST2 and PIIINP in the first $24 \mathrm{~h}$ of hospitalizations. All-cause mortality was registered for all patients after they were discharge over a median period of 339 days.

Results: In univariate Cox analysis, the three biomarkers were predictive of short-term mortality of ADHF patients. After adjusted for some clinical variables including age, admission systolic blood pressure, peripheral edema on admission, history of chronic obstructive pulmonary disease, admission sodium $<135 \mathrm{mmol} / \mathrm{L}$, admission hemoglobin, NT-proBNP, sST2 and PIIINP was significantly associated with the poor outcome (hazard ratio [HR] 1.32, 95\% confidence interval $[\mathrm{Cl}] 1.14-1.53, P<0.01 ; \mathrm{HR} 1.21,95 \% \mathrm{Cl} 1.03-1.43, P=0.020 ; \mathrm{HR} 1.40,95 \% \mathrm{Cl} 1.08-1.81, P=0.011)$. After added with Log2 PIIINP, but not Log2 sST2, the area under the curves (AUC) in the model of clinical variables and Log2 NT-proBNP could increase from 0.79 to 0.85 (95\% Cl 0.0071-0.10, $P=0.024)$. Furthermore, compared with the model of clinical variables, Log2 NT-proBNP, the improvement in the prognostic model of clinical variables, Log2 NTproBNP and Log2 PIIINP had statistical significance [net reclassification improvement (NRI) 0.31, $P=0.018$; integrated discrimination improvement (IDI) $0.068, P<0.01]$.
\end{abstract}

Conclusions: NT-proBNP, sST2 and PIIINP are independent prognostic factors for all-cause mortality in ADHF patients. Furthermore, the combination of NT-proBNP and PIIINP may provide incremental prognostic value over NT-proBNP in the survival of ADHF patients.

Keywords: Heart failure, Myocardial fibrosis, Biomarkers, Prognosis

*Correspondence: fenglizssrmyy@126.com

${ }^{1}$ Department of Cardiology, Zhongshan People Hospital, Sun Yat-Sen University, No. 2 Sunwen East Road, Zhongshan 528400, Guangdong, People's Republic of China

Full list of author information is available at the end of the article

\begin{abstract}
Introduction
During the past decade, acutely decompensated heart failure (ADHF), a terminal stage of heart disease characterized by high mortality, has become more and more prevalent, which creates a great burden for health care [1]. Identifying ADHF patients with high mortality risk and implementing effective cardiovascular preventive
\end{abstract}


measures successfully is therefore of great significance for improving those patients' survival. As one of the remarkable neurohormones from the heart, $\mathrm{N}$-terminal pro-B-type natriuretic peptide (NT-proBNP) is by far an effective indicator that have been verified for its role in predicting the outcomes in both ADHF and chronic heart failure (HF) [2-4], while its specificity and sensitivity remain low somehow $[5,6]$, which warrants a ADHF predictor with higher specificity and sensitivity.

Myocardial fibrosis, a central role in the formation and development of ADHF, was shown to be associated with cardiovascular outcomes. Furthermore, multiple researches have demonstrated the biomarkers of myocardial fibrosis, such as soluble suppression of tumorigenicity 2 (sST2) and Procollagen Type III N-Terminal Peptid (PIIINP), could serve as predictive factors to identify all-cause death in patients with HF [7-9]. Theoretically, heavier myocardial fibrosis in patients with heart failure means more severe ventricular remodeling of patients. Moreover, severe ventricular remodeling usually associates with poor outcomes in ADHF patients. Thus, it is possible that NT-proBNP combining with biomarkers of myocardial fibrosis would improve the accuracy of NT-proBNP in predicting major adverse outcomes [10]. However, no research before has focused on the combined value of sST2, PIIINP and NT-proBNP in identifying all-cause death in ADHF patients.

The current study was aimed to investigate whether the combination of sST2, PIIINP and NT-proBNP was more accurate than NT-proBNP alone in predicting short-term survival in patients with ADHF.

\section{Methods}

\section{Study population and design}

Between November 2018 and August 2019, 217 consecutive patients with ADHF were enrolled prospectively in Zhongshan People's Hospital, Guangdong Province, China. According to the related guidelines [2, 3], those patients in the study should have the signs and symptoms which indicated they had an attack of new-onset HF or acutely decompensated chronic HF (e.g., dyspnea, edema, weight gain). What's more, they should be observed with elevated levels of NT-proBNP and the impaired systolic or diastolic function of heart by echocardiography. Each patient' clinical data would be collected by two cardiologists within the first $24 \mathrm{~h}$ of the admission, including age, gender, body-mass index (BMI), systolic blood pressure (SBP), heart rate, comorbidities and New York Heart Association (NYHA) class. Furthermore, the results of laboratory examination such as Hemoglobin $(\mathrm{Hb})$, Serum Sodium, Serum Creatinine (Scr), Low-Density Lipoprotein Cholesterol (LDL-c), Hemoglobin A1c (HbA1c) and Procalcitonin (PCT) would be recorded; and Left ventricular ejection fraction (LVEF) was tested with Simpson's method. The blood sample would be collected within the first $24 \mathrm{~h}$ when they were on admission for the measurement of the levels of NT-proBNP, sST2 and PIIINP. In order to avoid the interferences in the biomarkers of myocardial fibrosis concentrations from other diseases, those who presented severe extracardial condition would not be included such as pulmonary interstitial fibrosis, metabolic bone diseases, liver diseases, or renal failure. Other exclusion criteria were age $<18$ years, acute coronary syndrome, unstable hemodynamics, cardiac surgery and malignant tumor.

The follow-up period would start from the first day when participants were discharged. The subjects would be investigated by the cardiologists who did not know about the results of the biomarkers. All the patients were treated with standard HF management according to the current clinical practice guidelines, and no one was observed to quit the study during the follow-up period. Data of all-cause mortality was collected with the means of regular telephone interviews, administrative databases and medical records monthly.

The study was performed in accordance with the Declaration of Helsinki, and approved by the local Regional Ethics Committee. All the participants in the study had signed written informed consent already.

\section{Biochemical measurement}

Blood samples would be centrifuged at $+4{ }^{\circ} \mathrm{C}$ after collection immediately and stored at $-80{ }^{\circ} \mathrm{C}$ at the laboratory until analysis. Serum sST2 were measured with human ST2 Quantikine ELISA Kit (R\&D Systems, Inc., Minneapolis, Minnesota, United States), in which the lower limit of detection is $0.005 \mathrm{ng} / \mathrm{ml}$ and measurement range is up to $2 \mathrm{ng} / \mathrm{ml}$. The inter- and intra-assay coefficients of variation were $4.4-5.6 \%$ and $5.4-7.1 \%$, respectively [11]. Now the risk threshold of sST2 in predicting all-cause death is still not validated enough accurately, and the range between 35 and $65 \mathrm{ng} / \mathrm{ml}$ was proposed by some studies according to Youden Index [12-15]. Serum PIIINP was measured with the Chemiluminescent Microparticle Immunoassay (AnTu Biological Co., Zhengzhou, China). The range of detection is $3.0-100.0 \mathrm{ng} / \mathrm{ml}$. The coefficients of variation were $<15.0 \%$. Serum NT-proBNP was tested by electrochemiluminescence immunoassay (Roche, Basel, Switzerland), and the upper limit of normal for NT-proBNP in the acute setting is $<300 \mathrm{pg} / \mathrm{ml}$.

\section{Statistical analysis}

The median \pm standard deviation or median and interquartile range was presented to describe the basic characters for continuous variables, and the percentage for categorical variables. For those continuous variables with 
a normal distribution and equal variance, the independent-sample student's T-test or ANOVA was used to compare the differences of two groups. Otherwise, rank sum test was used. Chi-square tests were chosen to evaluate the statistic significances between different groups in categorical variables. During the follow-up, all-cause death in ADHF patients was seen as the endpoint in the study.

2Log transformations were made for the three biomarkers in the following analysis because of their skewed distribution. Cox proportional hazards regression was conducted to perform the association between the biomarkers and mortality in ADHF patients. Univariable Cox regression analysis would study weather NTproBNP, sST2, PIIINP and other clinical variables were related to the all-cause death of ADHF patients, respectively. Adjusted by several clinical variables with utility of prognostic value in ADHF patients, the three biomarkers above were evaluated in the bootstrapped multivariable Cox regression analysis with Stepwise method. The 2log-transformed biomarkers with prognostic information would be added into clinical variables only model to build new models. Built the receiver operating characteristic (ROC) curves of the models, and calculated the area under the curves (AUC), respectively. The comparisons between models by a pair-wise method were performed with the method of Hanley JA and McNeil BJ [16]. What's more, net reclassification improvement (NRI) and absolute integrated discrimination index (IDI) would be tested to evaluate the improvement in predictive accuracy between the models by a pair-wise method.

When a $P$-value $<0.05$, it was considered statistically significant. PASW statistics version 24.0 (SPSS Inc., Chicago, IL, USA), MedCalc statistical software version 19.0.7 and $\mathrm{R}$ version 4.0.2 (http://www.r-project.org) were used to analyze the data attained in the study.

\section{Results}

\section{Patient characteristics}

The baseline characteristics of ADHF patients enrolled were showed in Table 1. The mean age of subjects was $66.18 \pm 13.60$ years and male $64.98 \% .167$ subjects (76.96\%) had NYHA class III or IV prior to decompensation and 127 subjects (58.53\%) peripheral edema on admission. The mean LVEF was $45.00 \%$ (32.00, 58.75). Comorbidities were: hypertension (57.14\%), coronary artery disease (45.16\%), atrial fibrillation (39.17\%), Diabetes mellitus (27.65\%) and chronic obstructive pulmonary disease $(10.60 \%)$. The mean concentration of sST2 is $19.63 \mathrm{ng} / \mathrm{ml}(12.61,35.53)$, PIIINP $6.70 \mathrm{ng} / \mathrm{ml}(4.84$, 9.96), and NT-proBNP $4491.00 \mathrm{pg} / \mathrm{ml}$ (1814.50, 8436.00). While the ADHF patients were treated with heart failure management, $84.79 \%$ of them used angiotensin-converting enzyme inhibitor or angiotensin-receptor blocker,
Table 1 Baseline characteristics of ADHF patients $(n=217)$

\begin{tabular}{|c|c|}
\hline Age (years) & $66.18 \pm 13.60$ \\
\hline Male, n (\%) & $141(64.98)$ \\
\hline BMI $\left(\mathrm{kg} / \mathrm{m}^{2}\right)$ & $24.82 \pm 3.65$ \\
\hline $\mathrm{SBP}(\mathrm{mmHg})$ & $141.22 \pm 24.99$ \\
\hline Heart rate (bmp) & $91.61 \pm 23.46$ \\
\hline Prior NYHA class I-II/III-IV, n (\%) & $50 / 167(23.04 / 76.96)$ \\
\hline Peripheral edema, n (\%) & $127(58.53)$ \\
\hline Hypertension, n (\%) & $124(57.14)$ \\
\hline CAD, n (\%) & $98(45.16)$ \\
\hline$A F, n(\%)$ & $85(39.17)$ \\
\hline Diabetes mellitus, n (\%) & $60(27.65)$ \\
\hline COPD, n (\%) & $23(10.60)$ \\
\hline Hemoglobin (g/dl) & $12.69 \pm 2.18$ \\
\hline Sodium < 135 mmol/L, n (\%) & $14(6.45)$ \\
\hline $\mathrm{SCr}$ (umol/L) & $94.00(79.00,120.50)$ \\
\hline $\mathrm{LDL}-\mathrm{c}(\mathrm{mmol} / \mathrm{L})$ & $2.45(1.73,3.15)$ \\
\hline $\mathrm{HbA1c}(\%)$ & $6.00(5.70,7.00)$ \\
\hline PCT (pg/ml) & $0.064(0.038,0.12)$ \\
\hline sST2 (ng/ml) & $19.63(12.61,35.53)$ \\
\hline PIIINP (ng/ml) & $6.70(4.84,9.96)$ \\
\hline NT-proBNP (pg/ml) & $4491.00(1814.50,8436.00)$ \\
\hline $\operatorname{LVEF}(\%)$ & $45.00(32.00,58.75)$ \\
\hline ACEl/ARB, n (\%) & $184(84.79)$ \\
\hline $\beta$-blocker, n (\%) & $157(72.35)$ \\
\hline Loop diuretic, n (\%) & $202(93.09)$ \\
\hline Aldosterone antagonist, n (\%) & $189(87.10)$ \\
\hline
\end{tabular}

$A C E I$, angiotensin-converting enzyme inhibitor; $A F$, atrial fibrillation; $A R B$, angiotensin-receptor blocker; BMI, body-mass index; CAD, coronary artery disease; COPD, chronic obstructive pulmonary disease; HbA1c, Hemoglobin A1c; LDL-c, low-density lipoprotein cholesterol; LVEF, left ventricular ejection fraction; NT-proBNP, N-terminal pro B-type natriuretic peptide; NYHA, New York Heart Association; $\mathrm{PCT}$, procalcitonin; PIIINP, procollagen type III N-terminal peptid; $\mathrm{SBP}$, systolic blood pressure; $\mathrm{SCr}$, serum creatinine; $\mathrm{SST} 2$, soluble suppression of tumorigenicity-2

72.35\% $\beta$ - blocker, 93.09\% Loop diuretic, and $87.10 \%$ Aldosterone antagonist.

\section{The biomarkers and the results of the survival analysis for all-cause mortality in ADHF patients}

37 patients $(17.05 \%)$ had died during the follow-up (median follow-up period was 339 days $[249,402])$. Compared with those alive at discharge, patients who died after going home from hospital were older, and the levels of admission NYHA class were higher; however, LVEF between two groups was of no statistical significance $[50.00(32.50,56.00)$ vs $44.00(32.00,60.00), P=0.91]$. The levels of NT-proBNP, sST2, and PIIINP among the population that died are higher than the others (pg/ $\mathrm{ml}, 8481.00(5064.00,13,882.50)$ vs 3947.50 (1644.75, 7580.50), $P<0.01 ; \mathrm{ng} / \mathrm{ml}, 28.56(15.91,61.22)$ vs 19.03 (11.91, 32.12), $P=0.010 ; \mathrm{ng} / \mathrm{ml}, 9.22(5.00,11.68)$ vs 6.14 
Table 2 Univariate and bootstrapped multivariate Cox regression analysis for all-cause mortality

\begin{tabular}{lllllll}
\hline Variables & Univariable & & & Multivariate & \\
\cline { 2 - 3 } \cline { 6 - 7 } & HR $(\mathbf{9 5 \%} \mathrm{Cl})$ & $\boldsymbol{P}$ & & HR (95\% Cl) & $\mathbf{P}$ \\
\hline Log2 SST2 & $1.23(1.06-1.44)$ & $<\mathbf{0 . 0 1}$ & & $1.21(1.03-1.43)$ & $\mathbf{0 . 0 2 0}$ \\
Log2 PIIINP & $1.38(1.11-1.71)$ & $<\mathbf{0 . 0 1}$ & & $1.40(1.08-1.81)$ & $\mathbf{0 . 0 1 1}$ \\
Log2 NT-proBNP & $1.34(1.17-1.54)$ & $<\mathbf{0 . 0 1}$ & & $1.32(1.14-1.53)$ & $<\mathbf{0 . 0 1}$ \\
\hline
\end{tabular}

Bold indicates the siginificant values $(P<0.05)$

$\mathrm{HR}$, hazard rate; $\mathrm{Cl}$, confidence interval

(4.55, 8.71), $P<0.01)$. For Diabetes patients, those who did not survive had higher concentration of the NTproBNP compared with those alive [10124.50 (4211.50, $20,064.00)$ vs 3330.50 (1682.25, 6547.75), $P=0.013$ ].

The Univariate Cox regression analysis showed sST2, PIIINP and NT-proBNP to be predictive of all-cause mortality $(P<0.01$, respectively) (Table 2$)$. Some clinical variables have been validated with their prognostic utility for all-cause mortality of patients with ADHF; therefore, they were made to a clinical adjustment model for determining if the 3 biomarkers had an independent value [age (per 10 years), admission systolic blood pressure (per $10 \mathrm{~mm} \mathrm{Hg}$ ), peripheral edema on admission, history of chronic obstructive pulmonary disease, admission sodium $<135 \mathrm{mmol} / \mathrm{L}$, admission hemoglobin] [1719]. After adjusted with the clinical variables above, the results from bootstrapped multivariate Cox regression analysis indicated that sST2, PIIINP and NT-proBNP were the prognostic factors of all-cause mortality independently $\left(\mathrm{HR}_{2 \log } 1.21,95 \% \mathrm{CI} 1.03-1.43, P=0.020\right.$; $\mathrm{HR}_{2 \log } 1.40,95 \% \mathrm{CI} 1.08-1.81, P=0.011 ; \mathrm{HR}_{2 \log } 1.32,95 \%$ CI 1.14-1.53, $P<0.01$ ) (Table 2).

Model 1 consisted of the six clinical factors above. Model 2 was made up of Model 1 and Log2 NT-proBNP. Model 3 was constructed by adding Model 2 and Log2 sST2, and Model 4 by adding Model 2 and Log2 PIIINP. Model 5 was built by combining Model 2 with Log2 sST2 and Log2 PIIINP. Table 3 showed the ROC curves of models for predicting the all-cause mortality of ADHF patients, in which the AUC of Model 1, 2, 3, 4 and 5 were 0.77 (95\% CI 0.69-0.84), 0.79 (95\% CI 0.71-0.86), 0.80 (95\% CI $0.72-0.86$ ), 0.85 (95\% CI $0.77-0.90$ ) and 0.85 (95\% CI 0.77-0.90), respectively (all $P<0.01$ ). Among them, the area under the curves of Model 4 performed better than that of clinical variables only model (Model 1), Model 2 and Model 3, respectively ( $\triangle$ AUC $0.076,95 \%$ CI $0.0044-0.15, P=0.038 ; \triangle A U C 0.054,95 \%$ CI $0.0071-$ $0.10, P=0.024 ; \triangle$ AUC $0.050,95 \%$ CI $0.00048-0.099$, $P=0.048$ ) (Table 4). More importantly, the comparisons of the area under the curves between Model 5, which contained additional Log2 sST2 compared with Model 4,
Table 3 The AUC of the ROC curves from different models and the comparisons

\begin{tabular}{llllll}
\hline & AUC & $\mathbf{9 5 \%} \mathbf{C l}$ & $\boldsymbol{P}$ & Sensitivity & Specificity \\
\hline Model 1 & 0.77 & $0.69-0.84$ & $<\mathbf{0 . 0 1}$ & 0.69 & 0.75 \\
Model 2 & 0.79 & $0.71-0.86$ & $<\mathbf{0 . 0 1}$ & 0.61 & 0.87 \\
Model 3 & 0.80 & $0.72-0.86$ & $<\mathbf{0 . 0 1}$ & 0.92 & 0.61 \\
Model 4 & 0.85 & $0.77-0.90$ & $<\mathbf{0 . 0 1}$ & 0.94 & 0.63 \\
Model 5 & 0.85 & $0.77-0.90$ & $<\mathbf{0 . 0 1}$ & 0.94 & 0.63 \\
\hline
\end{tabular}

Bold indicates the siginificant values $(P<0.05)$

Model 1: Clinical factors contained age, gender, NYHA class, AF, SCr and LVEF; Model 2: Model 1 + Log2 NT-proBNP; Model 3: Model 2+Log2 sST2; Model 4: Model $2+$ Log2 PIIINP; Model 5: Model $2+\log 2$ sST2 + Log2 PIIINP

AUC, area under curve; $\mathrm{Cl}$, confidence interval

and the three models above also got statistically significant results; however, Model 4 and Model 5 performed so similar that they could be approximately the same in predicting the death in ADHF patients $(\triangle \mathrm{AUC}<0.0010$, $P=1.00$ ) (Table 4). Besides, for ADHF patients, the prognosis of all-cause mortality had made no significant difference between Model 3 added with Log_sST2 and Model 2 added with Log2 NT-proBNP ( $\triangle \mathrm{AUC} 0.016$, $95 \% \mathrm{CI}-0.011$ to $0.043, P=0.25$ ) (Table 4). More details about the different ROC curves of models were shown in Table 4.

NRI and IDI were used to evaluate the improvement of predictive Models for all-cause mortality in ADHF patients (Table 5). Adding Log2 PIIINP instead of Log2 SST2 to the model of clinical risk factors and Log2 NTproBNP (Model 2) and forming Model 4 could make the NRI improve 0.31 (95\% CI 0.053-0.56, $P=0.018$ ) and IDI improve 0.068 (95\% CI 0.024-0.11, $P<0.01$ ). Simultaneously, the improvement of NRI and IDI would be validated in the setting of Model 5 in which Log2 PIIINP and Log2 sST2 were added to Model 2 (NRI: 0.31 improvement, $P=0.018$; IDI: 0.068 improvement, $P<0.01)$. It was worth noting that there was no statistically improvement in neither NRI nor IDI when Model 5 was compared with Model 4, even though there was the additional independent predictor Log2 sST2 in Model 5. The results above suggested that combining with PIIINP could make the model of NT-proBNP and clinical variables predict ADHF patients' mortality better. Remarkably, the addition of PIIINP and sST2 did not perform better than the addition of PIIINP alone for the model of NT-proBNP and clinical variables in predicting ADHF patients' death.

\section{Discussion}

The current study found sST2, PIIINP and NT-proBNP were respectively three independent risk factors for short-term all-cause death in patients with ADHF, and 
Table 4 The comparisons of the ROC curves between two different models

\begin{tabular}{|c|c|c|c|c|c|c|}
\hline & $\triangle A U C$ & $95 \% \mathrm{Cl}$ & $P$ & $\triangle \mathrm{AUC}$ & $95 \% \mathrm{Cl}$ & $P$ \\
\hline Compared to Model 1 & & & & Compared to Model 2 & & \\
\hline Model 2 & 0.060 & -0.022 to 0.14 & 0.15 & - & - & - \\
\hline Model 3 & 0.073 & -0.0082 to 0.16 & 0.078 & 0.016 & -0.011 to 0.043 & 0.25 \\
\hline Model 4 & 0.076 & $0.0044-0.15$ & 0.038 & 0.054 & $0.0071-0.10$ & 0.024 \\
\hline Model 5 & 0.076 & $0.0044-0.15$ & 0.038 & 0.054 & $0.0071-0.10$ & 0.024 \\
\hline Compared to Model 3 & & & & Compared to Model 4 & & \\
\hline Model 4 & 0.050 & $0.00048-0.099$ & 0.048 & - & - & - \\
\hline Model 5 & 0.050 & $0.00048-0.099$ & 0.048 & 0.00 & - & 1.0 \\
\hline
\end{tabular}

Bold indicates the siginificant values $(P<0.05)$

Model 1: Clinical factors contained age, gender, NYHA class, AF, SCr and LVEF; Model 2: Model 1+Log2 NT-proBNP; Model 3: Model 2 + Log2 sST2; Model 4: Model 2+ Log2 PIIINP; Model 5: Model 2+ Log2 SST2 + Log2 PIIINP

$\mathrm{Cl}$, confidence interval

Table 5 Predictive improvement in the models for all-cause mortality

\begin{tabular}{|c|c|c|c|c|c|c|}
\hline Model & NRI & $95 \% \mathrm{Cl}$ & $P$ & IDI & $95 \% \mathrm{Cl}$ & $P$ \\
\hline Model 2 & $0.68^{*}$ & $0.36-1.01$ & $<0.01$ & $0.12^{*}$ & $0.056-0.18$ & $<0.01$ \\
\hline \multirow[t]{2}{*}{ Model 3} & $0.64^{*}$ & $0.31-0.97$ & $<0.01$ & $0.14^{*}$ & $0.072-0.21$ & $<0.01$ \\
\hline & $0.10^{* *}$ & -0.25 to 0.46 & 0.56 & $0.021^{* *}$ & -0.0042 to 0.046 & 0.10 \\
\hline \multirow[t]{3}{*}{ Model 4} & $0.52^{*}$ & $0.20-0.84$ & $<0.01$ & $0.17^{*}$ & $0.10-0.24$ & $<0.01$ \\
\hline & $0.31^{* *}$ & $0.053-0.56$ & 0.018 & $0.068^{* *}$ & $0.024-0.11$ & $<0.01$ \\
\hline & $0.20^{* * *}$ & -0.12 to 0.52 & 0.23 & $0.054^{* * *}$ & $0.0065-0.10$ & 0.026 \\
\hline \multirow[t]{4}{*}{ Model 5} & $0.52^{*}$ & $0.20-0.84$ & $<0.01$ & $0.17^{*}$ & $0.10-0.24$ & $<0.01$ \\
\hline & $0.31^{* *}$ & $0.053-0.56$ & 0.018 & $0.068^{* *}$ & $0.024-0.11$ & $<0.01$ \\
\hline & $0.20^{* * *}$ & -0.12 to 0.52 & 0.23 & $0.054^{* * *}$ & $0.0068-0.10$ & 0.025 \\
\hline & $0.011^{* * * *}$ & -0.36 to 0.40 & 0.95 & 0.00 & - & 1.0 \\
\hline
\end{tabular}

Bold indicates the siginificant values $(P<0.05)$

Model 1: Clinical factors contained age, gender, NYHA class, AF, SCr and LVEF; Model 2: Model 1+ Log2 NT-proBNP; Model 3: Model $2+$ Log2 sST2; Model 4: Model

2+ Log2 PIIINP; Model 5: Model 2+ Log2 sST2 + Log2 PIIINP

$\mathrm{Cl}$, confidence interval; NRI, net reclassification improvement; IDI, integrated discrimination improvement

${ }^{*}$ Compared with Model $1 ;{ }^{* *}$ compared with Model 2; ${ }^{* * *}$ Compared with Model $3 ;{ }^{* * * *}$ compared with Model 4

higher levels of these biomarkers were associated with higher risk of all-cause death. More importantly, PIIINP could improve risk stratification with NT-proBNP in predicting short-term survival among ADHF patients.

Multiple studies have shown higher NT-proBNP level was associated with higher incidence of poor clinical outcomes, including heart failure readmission, cardiovascular mortality, all-cause mortality, and composite outcomes in ADHF patients. Data from Mario Petretta et al. demonstrated that NT-proBNP levels may differentiate chronic heart failure patients with a higher risk of cardiac mortality [4]. The study from Santaguida PL et al. showed that NT-proBNP was an independent predictor of all-cause and cardiovascular mortality in ADHF [20-22]. In addition, Savarese G and the colleagues found that higher level of NT-proBNP could predict higher risk of HF-related admission [23]. Based on these studies, NT-proBNP was recommended by current guidelines as a prognostic marker in ADHF patients [2, 3]. Diabetics was demonstrated as the risk factor to affect clinical outcomes in HF [24], and among them the high concentrations of NT-proBNP was associated with high rate of rehospitalization for acutely heart failure [24]. Consistently, the current study found that ADHF patients with increased NT-proBNP level were associated with worse outcomes than those without. However, caution should be noted that the level of the biomarker in patients could be influenced by a wide variety of factors including age, weight, renal function, and noncardiac diseases $[5,6]$. Simultaneously, our study have demonstrated that NTproBNP could not provide incremental prognostic value significantly after added to clinical variables only model 
according to the comparison of AUC between two models (Model 1 and Model 2) in the study, indicating that strategies combining other biomarkers may inform clinicians to make further NT-proBNP-based risk stratification in ADHF patients.

PIIINP, one of the biomarkers of myocardial fibrosis studied in the current study, was observed to be deposited in cardiac extracellular matrix during the development of cardiac remodeling, resulting in worse ventricular compliance as well as poor outcomes [25]. In fact, PIIINP could also be regarded as the surrogate of myocardial fibrosis. It was reported that high level of circulating PIIINP was predictive of increased allcause mortality and HF readmission risk among African Americans with chronic HF [9]. Similarly, the data shown in the study demonstrated that high level of PIIINP was strongly linked to the incidence of all-cause death in patients with ADHF, no matter adjusting or not adjusting confounding factors, further suggesting that it was an emerging prognostic factors independently for all-cause mortality among ADHF patients.

Considering this, the current study firstly combined NT-proBNP with PIIINP and intended to improve the accuracy of survival prediction in ADHF patients. Our data showed the combination of NT-proBNP and PIIINP was significantly more effective than NT-proBNP alone in predicting all-cause death in patients with ADHF. After adding Log2 PIIINP to the model of Log2 NTproBNP and clinical variables, the sensitivity of the new model (Model 4) in predicting ADHF patients' survival could be up to 0.94 , which would invariably accord with the statistically significant improvement of AUC, NRI, and IDI. In addition to NT-proBNP, the data comparing the combination of two biomarkers generated from different pathological mechanism (NT-proBNP and PIIINP) and NT-proBNP alone have demonstrated the role of PIIINP in ADHF patients screening to detect those who would survive in the short-term follow-up should be valued in clinical practice. In fact, with regard to the reason for the increased prognostic information brought by PIIINP on the basis of NT-proBNP in ADHF patients, the severity of HF symptoms and myocardial remodeling should be focused on. Theoretically, ADHF patients with poor survival usually accompany with increased levels of myocardial remodeling and fibrosis. In other words, with low level of myocardial fibrosis, those patients are more likely to survive during the follow-up. Therefore, as one of biomarkers of myocardial fibrosis, low PIIINP values are linked to early stage of myocardial and matrix remodeling and, sometimes possibly, means the cardiac remodeling has not even existed in HF progression, resulting in an efficient cardiac function and an acceptable clinical outcome in ADHF patients. Notably, our predictive model of NT-proBNP combined with PIIINP not only took into account the disorder of cardiac neuroendocrine function in patients with ADHF, but also considered the structural disorders of cardiac remodeling, thus was more sensitive than NT-proBNP alone in predicting short-term all-cause mortality. In short, the current study firstly came out with a survival model of NT-proBNP combined with PIIINP, and found that the combination of the two biomarkers could perform better than NTproBNP alone to rule out for the prognosis of short-term all-cause mortality in ADHF patients.

sST2 is a common biomarker of myocardial fibrosis, whose increase was associated with higher risks of HF adverse events [26-28]. Previous researches have demonstrated the predictive value of SST2 for HF patients, in which ADHF patients with higher level of admission sST2 suffered higher risk of all-cause mortality and cardiovascular death than those without. The current study added some new evidence into this area by demonstrating that circulating SST2 value in ADHF patients could be a strong independent predictor of short term all-cause death. However, compared with PIIINP, sST2 showed nonsignificant incremental information on the basis of prognostic model of NT-proBNP and clinical variables in patients with ADHF. In fact, although both sST2 and PIIINP could be used to evaluate the degree of myocardial remodeling, the former awaits more available clinical evidence to validate the association between it and myocardial fibrosis histologically compared to the latter [25, $29,30]$, which may indicate the biomarker is less accurate than PIIINP in assessing myocardial remodeling in ADHF patients. In fact, as the members of the IL-1 receptor-like family of proteins, the resource of sST2 is still indefinite [31-33]. In the study, the sST2 values could not provide more prognostic information after adding to the model of NT-proBNP, PIIINP and clinical variables than before in ADHF patients' survival, suggesting that the combination of NT-proBNP and two biomarkers of fibrosis may not be more effective than that of NT-proBNP and PIIINP.

However, several limitations should be noted when interpreting. Firstly, the current study was performed on single center with relative small sample size, the conclusion of which still warrants confirmed in multiple centers with larger sample size. Nevertheless, the current study has validated the models built by bootstrapping internally. Besides, the multi-biomarker approach consistently showed a positive result in model performance when considering confounding factors of clinical variables, further demonstrating a survival diagnosis improvement. Secondly, previous studies found that changes of NT-proBNP, sST2 and PIIINP levels during follow-up were also shown to be associated with the prognosis of $\mathrm{HF}$ respectively $[34,35]$, while the changes were not 
considered in this study because all the circulating biomarkers were tested only once when discharged.

\section{Conclusions}

The current study suggested that high levels of NTproBNP, sST2 and PIIINP were associated with increased mortality in ADHF patients. Furthermore, PIIINP could take complementary role for risk stratification in all-cause mortality among ADHF patients next to NT-proBNP.

\begin{abstract}
Abbreviations
ACEl: Angiotensin-converting enzyme inhibitor; ADHF: Acutely decompensated heart failure; ARB: Angiotensin-receptor blocker; AUC: Area under the curves; BMI: Body-mass index; Cl: Confidence interval; COPD: Chronic obstructive pulmonary disease; Hb: Hemoglobin; HbA1c: Hemoglobin A1c; HF: Heart failure; HR: Hazard ratio; IDI: Integrated discrimination index; LDL-C: Low-density lipoprotein cholesterol; LVEF: Left ventricular ejection fraction; NT-proBNP: N-terminal pro-B-type natriuretic peptide; NRI: Net reclassification improvement; NYHA: New York Heart Association; PCT: Procalcitonin; PIIINP: Procollagen type III N-terminal peptid; SBP: Systolic blood pressure; SCr: Serum creatinine; sST2: Soluble suppression of tumorigenicity-2.
\end{abstract}

\section{Acknowledgements}

We thank all the people participating in the current study.

\section{Authors' contributions}

Design of the study: LF, YX, YY, JS. Material preparation and data collection: YY, YS, BH. Data analysis: YY, YS. Manuscript draft: YY, SW. All authors read and approved the final manuscript.

\section{Funding}

This study was not supported by any funding.

\section{Availability of data and materials}

The datasets generated and analyzed during the current study are not publicly available due to the consent given by study participants but are available from the corresponding author on reasonable request.

\section{Declarations}

\section{Ethics approval and consent to participate}

The study was performed in accordance with the Declaration of Helsinki. This study was approved by the Ethics Committee of Zhongshan People Hospital, Sun Yet-san University. All the participants in the study had signed written informed consent.

\section{Consent for publication}

Not applicable.

\section{Competing interests}

The authors declare that they have no competing interests.

\begin{abstract}
Author details
${ }^{1}$ Department of Cardiology, Zhongshan People Hospital, Sun Yat-Sen University, No. 2 Sunwen East Road, Zhongshan 528400, Guangdong, People's Republic of China. ${ }^{2}$ Department of Cardiology, State Key Laboratory of Organ Failure Research, Nanfang Hospital, Southern Medical University, Guangzhou, People's Republic of China.
\end{abstract}

Received: 27 February 2021 Accepted: 21 May 2021 Published online: 28 May 2021

\section{References}

1. Heidenreich PA, Albert NM, Allen LA, Bluemke DA, Butler J, Fonarow GC, et al. Forecasting the impact of heart failure in the United States: a policy statement from the American Heart Association. Circ Heart Fail. 2013;6:606-19.

2. Yancy CW, Jessup M, Bozkurt B, Butler J, Casey DE Jr, Colvin MM, et al. 2017 ACC/AHA/HFSA focused update of the 2013 ACCF/AHA guideline for the management of heart failure: a report of the American College of Cardiology/American Heart Association Task Force on Clinical Practice Guidelines and the Heart Failure Society of America. Circulation. 2017;136:e137-61.

3. Ponikowski P, Voors AA, Anker SD, Bueno H, Cleland JGF, Coats AJS, et al. ESC Guidelines for the diagnosis and treatment of acute and chronic heart failure: The Task Force for the diagnosis and treatment of acute and chronic heart failure of the European Society of Cardiology (ESC). Developed with the special contribution of the Heart Failure Association (HFA) of the ESC. Eur Heart J. 2016;37:2129-200.

4. Petretta M, Colao A, Sardu C, Scopacasa F, Marzullo P, Pivonello R, et al. NTproBNP, IGF-I and survival in patients with chronic heart failure. Growth Horm IGF Res. 2007;17:288-96.

5. Mehra MR, Uber PA, Park MH, Scott RL, Ventura HO, Harris BC, et al. Obesity and suppressed B-type natriuretic peptide levels in heart failure. J Am Coll Cardiol. 2004;43:1590-5.

6. McCullough PA, Duc P, Omland T, McCord J, Nowak RM, Hollander JE, et al. B-type natriuretic peptide and renal function in the diagnosis of heart failure: an analysis from the Breathing Not Properly Multinational Study. Am J Kidney Dis. 2003;41:571-9.

7. Pascual-Figal DA, Bayes-Genis A, Asensio-Lopez MC, Hernandez-Vicente A, Garrido-Bravo I, Pastor-Perez F, et al. The interleukin-1 axis and risk of death in patients with acutely decompensated heart failure. J Am Coll Cardiol. 2019:73:1016-25.

8. Morrow DA, Velazquez EJ, DeVore AD, Prescott MF, Duffy Cl, Gurmu $Y$, et al. Cardiovascular biomarkers in patients with acute decompensated heart failure randomized to sacubitril-valsartan or enalapril in the PIONEER-HF trial. Eur Heart J. 2019;40:3345-52.

9. Mansour IN, Bress AP, Groo V, Ismail S, Wu G, Patel SR, et al. Circulating procollagen type III N-terminal peptide and mortality risk in African Americans With heart failure. J Card Fail. 2016;22:692-9.

10. Pascual-Figal DA, Manzano-Fernández S, Boronat M, Casas T, Garrido IP, Bonaque JC, et al. Soluble ST2, high-sensitivity troponin T and N-terminal pro-B-type natriuretic peptide: complementary role for risk stratification in acutely decompensated heart failure. Eur J Heart Fail. 2011;13:718-25.

11. Dieplinger B, Januzzi JL Jr, Steinmair M, Gabriel C, Poelz W, Haltmayer M, et al. Analytical and clinical evaluation of a novel high-sensitivity assay for measurement of soluble ST2 in human plasma-the Presage ST2 assay. Clin Chim Acta. 2009;409:33-40.

12. Pascual-Figal DA, Bayes-Genis A, Asensio-Lopez MC, Hernández-Vicente A, Garrido-Bravo I, Pastor-Perez F, et al. The interleukin-1 axis and risk of death in patients with acutely decompensated heart failure. J Am Coll Cardiol. 2019;73:1016-25.

13. Parikh RH, Seliger SL, Christenson R, Gottdiener JS, Psaty BM, de Filippi CR. Soluble ST2 for prediction of heart failure and cardiovascular death in an elderly, community-dwelling population. J Am Heart Assoc. 2016;5:e003188.

14. Pascual-Figal DA, Manzano-Fernández S, Boronat M, Casas T, Garrido IP, Bonaque JC, et al. Soluble ST2, high-sensitivity troponin T- and N-terminal pro-B-type natriuretic peptide: complementary role for risk stratification in acutely decompensated heart failure. Eur J Heart Fail. 2011;13:718-25.

15. Rehman SU, Mueller T, Januzzi JL. Characteristics of the novel interleukin family biomarker ST2 in patients with acute heart failure. J Am Coll Cardiol. 2008;52:1458-65.

16. Hanley JA, McNeil BJ. The meaning and use of the area under a receiver operating characteristic (ROC) curve. Radiology. 1982;143:29-36.

17. Salah K, Kok WE, Eurlings LW, Bettencourt P, Pimenta JM, Metra M, et al. A novel discharge risk model for patients hospitalised for acute decompensated heart failure incorporating $\mathrm{N}$-terminal pro-B-type natriuretic peptide levels: a European coLlaboration on Acute decompeNsated Heart Failure: ÉLAN-HF Score. Heart. 2014;100:115-25.

18. Scrutinio D, Ammirati E, Guida P, Passantino A, Raimondo R, Guida V, et al. Clinical utility of $\mathrm{N}$-terminal pro-B-type natriuretic peptide for risk stratification of patients with acute decompensated heart failure. 
Derivation and validation of the ADHF/NT-proBNP risk score. Int J Cardiol. 2013;168:2120-6.

19. Kociol RD, Horton JR, Fonarow GC, Reyes EM, Shaw LK, O'Connor CM, et al. Admission, discharge, or change in B-type natriuretic peptide and long-term outcomes. Circul Heart Fail. 2011;4:628-36.

20. Volpe M, Rubattu S, Burnett J. Natriuretic peptides in cardiovascular diseases: current use and perspectives. Eur Heart J. 2014;35:419-25.

21. Santaguida PL, Don-Wauchope AC, Oremus M, McKelvie R, Ali U, Hill SA, et al. BNP and NT-proBNP as prognostic markers in persons with acute decompensated heart failure: a systematic review. Heart Fail Rev. 2014;19:453-70.

22. Pascual-Figal DA, Manzano-Fernandez S, Boronat M, Casas T, Garrido IP, Bonaque JC, et al. Soluble ST2, high-sensitivity troponin T- and N-terminal pro-B-type natriuretic peptide: complementary role for risk stratification in acutely decompensated heart failure. Eur J Heart Fail. 2011;13:718-25.

23. Savarese G, Musella F, D'Amore C, Vassallo E, Losco T, Gambardella F, et al. Changes of natriuretic peptides predict hospital admissions in patients with chronic heart failure: a meta-analysis. JACC Heart Fail. 2014;2:148-58.

24. Sardu C, Santamaria M, Funaro S, Sacra C, Barbieri M, Paolisso P, et al. Cardiac electrophysiological alterations and clinical response in cardiac resynchronization therapy with a defibrillator treated patients affected by metabolic syndrome. Medicine (Baltimore). 2017;96:e6558.

25. Klappacher G, Franzen P, Haab D, Mehrabi M, Binder M, Plesch K, et al. Measuring extracellular matrix turnover in the serum of patients with idiopathic or ischemic dilated cardiomyopathy and impact on diagnosis and prognosis. Am J Cardiol. 1995;75:913-8.

26. Weir RA, Miller AM, Murphy GE, Clements S, Steedman T, Connell JM, et al. Serum soluble ST2: a potential novel mediator in left ventricular and infarct remodeling after acute myocardial infarction. J Am Coll Cardiol. 2010;55:243-50.

27. Shah RV, Chen-Tournoux AA, Picard MH, van Kimmenade RR, Januzzi JL. Serum levels of the interleukin-1 receptor family member ST2, cardiac structure and function, and long-term mortality in patients with acute dyspnea. Circ Heart Fail. 2009;2:311-9.

28. Sardu C, Marfella R, Santamaria M, Papini S, Parisi Q, Sacra C, et al. Stretch, injury and inflammation markers evaluation to predict clinical outcomes after implantable cardioverter defibrillator therapy in heart failure patients with metabolic syndrome. Front Physiol. 2018;9:758.

29. Fabiani I, Conte L, Pugliese NR, Calogero E, Barletta V, Di Stefano R, et al. The integrated value of SST2 and global longitudinal strain in the early stratification of patients with severe aortic valve stenosis: a translational imaging approach. Int J Cardiovasc Imaging. 2017;33:1915-20.

30. Izawa H, Murohara T, Nagata K, Isobe S, Asano H, Amano T, et al. Mineralocorticoid receptor antagonism ameliorates left ventricular diastolic dysfunction and myocardial fibrosis in mildly symptomatic patients with idiopathic dilated cardiomyopathy: a pilot study. Circulation. 2005;112:2940-5.

31. Pascual-Figal DA, Pérez-Martínez MT, Asensio-Lopez MC, Sanchez-Más J, García-García ME, Martinez CM, et al. Pulmonary production of soluble ST2 in heart failure. Circ Heart Fail. 2018;11:e005488.

32. Lipsky BP, Toy DY, Swart DA, Smithgall MD, Smith D. Deletion of the ST2 proximal promoter disrupts fibroblast-specific expression but does not reduce the amount of soluble ST2 in circulation. Eur J Immunol. 2012:42:1863-9.

33. Bartunek J, Delrue L, Van Durme F, Muller O, Casselman F, De Wiest B, et al. Nonmyocardial production of ST2 protein in human hypertrophy and failure is related to diastolic load. J Am Coll Cardiol. 2008;52:2166-74.

34. McKelvie RS, Komajda M, McMurray J, Zile M, Ptaszynska A, Donovan M, et al. Baseline plasma NT-proBNP and clinical characteristics: results from the irbesartan in heart failure with preserved ejection fraction trial. J Card Fail. 2010;16:128-34.

35. Jhund PS, Anand IS, Komajda M, Claggett BL, McKelvie RS, Zile MR, et al. Changes in N-terminal pro-B-type natriuretic peptide levels and outcomes in heart failure with preser ved ejection fraction: an analysis of the I-Preserve study. Eur J Heart Fail. 2015;17:809-17.

\section{Publisher's Note}

Springer Nature remains neutral with regard to jurisdictional claims in published maps and institutional affiliations.
Ready to submit your research? Choose BMC and benefit from:

- fast, convenient online submission

- thorough peer review by experienced researchers in your field

- rapid publication on acceptance

- support for research data, including large and complex data types

- gold Open Access which fosters wider collaboration and increased citations

- maximum visibility for your research: over 100M website views per year

At BMC, research is always in progress.

Learn more biomedcentral.com/submissions 